\title{
CRISTAIS LÍQUIDOS COLESTÉRICOS: A QUIRALIDADE REVELA AS SUAS CORES
}

\author{
Fernando Ely ${ }^{*}$, Marcos H. Mamoru Otsuka Hamanaka e Alaide Pellegrini Mammana \\ Divisão de Mostradores de Informação, Centro de Pesquisas Renato Archer, Ministério da Ciência e Tecnologia, \\ Rodovia Dom Pedro I, km 143,6. 13069-901 Campinas - SP, Brasil
}

Recebido em 3/10/06; aceito em 15/3/07; publicado na web em 5/10/07

\begin{abstract}
CHOLESTERIC LIQUID CRSTALS: CHIRALITY SHOWS ITS COLORS. Chirality deeply impacts the macroscopic properties on all scales of the universe from elemental particles through biological structures up to astronomic systems. Specifically, chiral liquid crystals give rise to self-organized macrostructures with unparalleled properties. Here we present easy experiments with a special class of chiral liquid crystals that show temperature-induced selective reflection of light (thermochromic effect). Understanding this thermochromic effect allows undergraduate students better realize important properties of super and supramolecular systems.
\end{abstract}

Keywords: liquid crystals; chirality; thermochromic effect.

\section{INTRODUÇÃO}

Estruturas sem simetria especular são muito encontradas na natureza. A existência ou a falta de simetria especular exerce um papel importante em todas as escalas do universo, desde partículas elementares a estruturas químicas e biológicas e mesmo sistemas astronômi$\cos ^{1}$. A simetria ou assimetria pode ter grandes conseqüências, por exemplo, de duas diferentes estruturas moleculares (enantiomeros), uma pode ser uma droga útil e a outra, altamente tóxica. Em sistemas auto-organizados, como cristais líquidos, o impacto da presença da quiralidade é muito grande, produzindo macroestruturas com propriedades incomparáveis tais como, polarização espontânea ferroelétrica e reflexão seletiva da luz². Em função disso, boa parte da pesquisa em cristais líquidos está centrada em sistemas quirais, muito em função das aplicações tecnológicas associadas ${ }^{3}$ (por exemplo obturadores de luz, microdisplays ultra-rápidos, detectores piroelétricos etc.).

Um cristal líquido é um fluido ordenado intermediário entre a fase cristal, ordenada em três dimensões, e a fase líquida desordenada (Figura 1). Frequientemente, refere-se um cristal líquido como mesofase e suas moléculas constituintes como mesógenos. A vasta maioria dos mesógenos termotrópicos é caracterizada por compostos em forma de bastão constituídos de um núcleo aromático rígido e cadeias terminais alquílicas (cristais líquidos calamíticos). Esses materiais podem formar duas classes principais de fases líquido-cristalinas, as fases esmética e nemática. A fase nemática (N) é um fluido unidimensionalmente ordenado em que os longos eixos moleculares estão, na média, orientados ao longo de um vetor $\mathbf{n}$ (o diretor) ${ }^{4}$. Assim, o diretor representa a direção média das moléculas em uma determinada fase líquido-cristalina.

Dentre os diversos tipos de cristais líquidos conhecidos, os nemáticos (N) são os mais importantes, muito devido a sua baixa viscosidade e elevado tempo de resposta a impulsos elétricos, para a construção de mostradores de informação (displays). Assim, a maioria dos displays para computadores portáteis (laptops), pessoais e de bolso (palm-tops), telefones celulares, câmeras fotográficas digitais e televisores não emissivos de alta resolução (LCDTV) são construídos a partir de cristais líquidos nemáticos ${ }^{5}$.

$\mathrm{Na}$ situação mais comum, assimetria molecular impõe quiralidade

*e-mail: fernando.ely@cenpra.gov.br

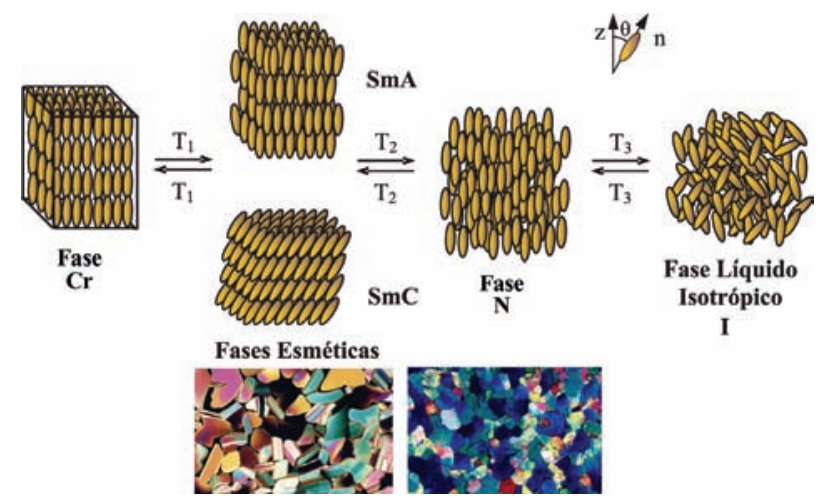

Figura 1. Transições de fase entre as fases cristal, cristal líquido e líquido isotrópico para um material calamítico como função da temperatura. Onde os elipsóides representam moléculas; $C r=$ cristal (sólido); $\operatorname{SmA}=$ fase líquidocristalina esmética $A ; \operatorname{SmC}=$ fase líquido-cristalina esmética $C ; N=$ fase líquido-cristalina nemática; $I$ = líquido isotrópico. Abaixo das figuras são apresentadas fotomicrografias (aumento 66x) de fases líquido-cristalinas esmética e nemática obsrvadas em microscópio óptico de luz plano polarizada

de forma às fases líquido-cristalinas, que é manifestada na formação do ordenamento helicoidal das moléculas constituintes da fase. Em especial, a introdução de quiralidade à fase nemática $(\mathrm{N})$ dá origem à fase nemática quiral ou colestérica $\left(\mathrm{N}^{*}\right)$. Nela as moléculas quirais empacotam para minimizar interações estéricas desfavoráveis e maximizar interações dipolares atrativas, formando uma macroestrutura helicoidal opticamente ativa (Figura 2).

Quando o passo da hélice (distância para uma revolução de $360^{\circ}$ ) formada pelo ordenamento orientacional das moléculas é comparável a faixa da luz visível, a fase helicoidal, espalhará ou refletirá luz irridiscente ${ }^{6}$. Ainda, o passo da hélice é sensível à temperatura, porque depende das orientações e interações das moléculas, que são também dependentes da temperatura. Portanto, a cor da luz espalhada também depende da temperatura ambiente, conseqüentemente sendo capaz de produzir um efeito termocrômico quando o passo da hélice é comparável ao comprimento de onda da luz. Além disso, estruturas helicoidais podem ser desenroladas (relaxadas) pela aplicação de um campo elétrico que acople com a polaridade ou 


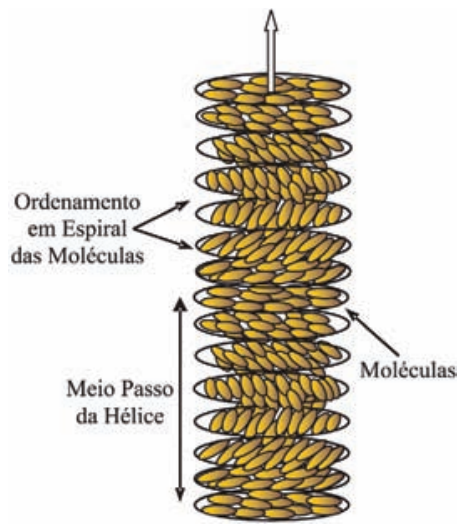

Figura 2. Macroestrutura helicoidal da fase colestérica ou nemática quiral $\left(N^{*}\right)$

anisotropia dielétrica do material. Assim, se a hélice está espalhando luz visível, isto pode ser evitado pela aplicação de um campo elétrico, dessa forma produzindo um efeito eletroóptico. Termômetros, espelhos polarizantes, mostradores eletroópticos, estocagem óptica de dados, pigmentos e guias de onda para transmissão de dados são algumas das aplicações já bem estabelecidas das estruturas helicoidais e polares de cristais líquidos sem simetria especular, tais como os colestéricos $\left(\mathrm{N}^{*}\right)^{1}$.

Nesse contexto, é objetivo desse trabalho demonstrar o efeito da quiralidade sobre sistemas auto-organizados usando como exemplo cristais líquidos colestéricos (nemáticos quirais). Esse efeito pode ser visualizado através das propriedades macroscópicas que se originam desses materiais orgânicos, em especial a reflexão seletiva da luz visível. Através dessa propriedade, serão preparadas diversas misturas de cristais líquidos cobrindo diferentes faixas de temperatura com conseqüente variação de cor e um adesivo capaz de mapear regiões de diferentes temperaturas em um objeto ou ser humano. Esses experimentos surgem, assim, como interessantes instrumentos para demonstrar para alunos de graduação e pós-graduação as propriedades macroscópicas dessas superestruturas moleculares.

\section{PARTE EXPERIMENTAL}

\section{Misturas líquido-cristalinas sensíveis à temperatura}

Misturas líquido-cristalinas com diferentes composições sensíveis à temperatura são preparadas. A Tabela 1 organiza as diversas composições de oleilcarbonato de colesteríla, pelargonato de colesteríla e benzoato de colesteríla (Figura 3) que produzem diferentes cores em diferentes temperaturas. Esses materiais estão disponíveis comercialmente na empresa Aldrich Chemical Company (a Merck - divisão de cristais líquidos também fornece esses materiais) a um custo aproximado de 8 reais/grama ou podem, ainda serem facilmente obtidos por esterificação do colesterol com os cloretos de ácidos adequados.

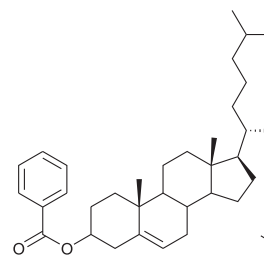

Benzoato de Colesterila
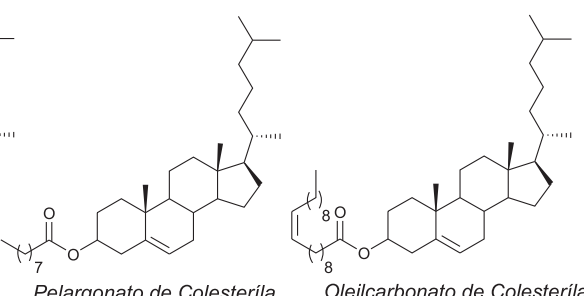

Figura 3. Estruturas químicas dos cristais líquidos colestéricos utilizados nos experimentos

\section{Procedimento 1}

a) Pese os materiais líquido-cristalinos na proporção de acordo com a Tabela 1 e transfira para pequenos frascos de vidro de 1 ou 3 $\mathrm{mL}$ com tampa. Benzoato de colesterila e o pelargonato de colesterila são sólidos a temperatura ambiente, podendo ser transferidos com um pequeno funil de vidro. O oleilcarbonato de colesterila tem um aspecto pastoso e é melhor transferido para o frasco com o auxílio de uma espátula. Tome cuidado para evitar perdas, pois isso alterará a proporção entre os componentes e, em decorrência, a faixa de temperatura em que o efeito termocrômico é observado será modificada.

b) Aqueça cuidadosamente (aproximadamente $100{ }^{\circ} \mathrm{C}$ ) os materiais sólidos nos frascos, usando uma pistola de aquecimento (soprador térmico) ou uma chapa de aquecimento. Uma mistura líquida incolor ou levemente amarelada é obtida após o aquecimento.

c) Com o resfriamento, as misturas líquidas tornam-se coloridas. A coloração aparece devido à mudança de fase do material, que passa do estado líquido para o estado cristal líquido. Composições diferentes fornecem cores diferentes sobre faixas de temperatura diferentes.

\section{Adesivo para mapear a temperatura ("adesivo do humor")}

\section{Procedimento 2}

a) Coloque sobre a mesa, limpa e lisa, um pedaço quadrado de papel contact ${ }^{\circledR}$ preto (aproximadamente $3 \mathrm{~cm}$ de lado) com a parte preta voltada para cima.

b) Com o auxílio de uma espátula coloque uma pequena porção da mistura líquido-cristalina 7 (Tabela 1) no centro do filme plástico (Figuras 4a e 4b).
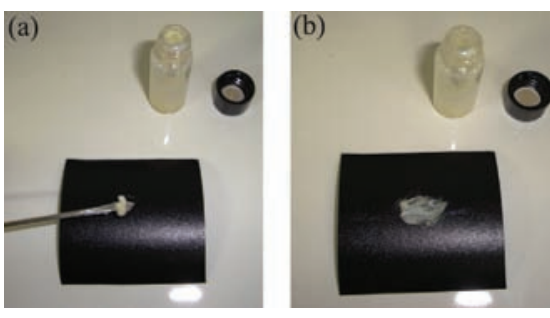

Figura 4. Transferência do material líquido-cristalino para o filme plástico contact $^{\circledR}$

c) Tome um pequeno quadrado (aproximadamente $2,5 \mathrm{~cm}$ de lado) de plástico de transparência para projeção limpa e translúcida (tampa). Coloque cuidadosamente sobre o fluido líquido-cristalino, de maneira a formar um "sanduíche" de cristal líquido (Figuras 5 a e $5 b)$.
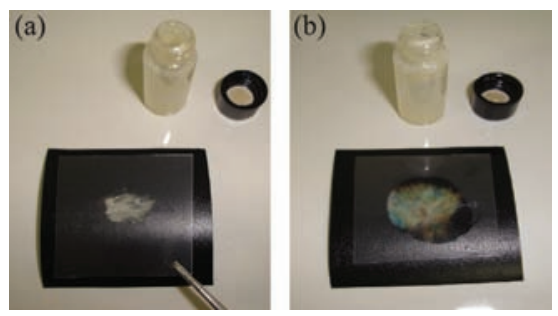

Figura 5. Colocação da tampa

d) Pressione cuidadosamente a tampa com o dedo. O calor do dedo aquece a mistura líquido-cristalina colestérica e faz o fluido espalharse, preenchendo a área abaixo da tampa. $\mathrm{O}$ objetivo aqui é distribuir uniformemente a camada de cristal líquido sem muitas bolhas. 
e) Retire um pedaço de fita adesiva (3M Scotch Super Strength $\AA$, $4,5 \mathrm{~cm}$ de largura) e cole cuidadosamente sobre o sanduíche base/ tampa de cristal líquido. Tente fazer isto de maneira a evitar ao máximo a formação de bolhas de ar. Aplique pressão para suavizar a fita sobre o sanduíche, selando-o sobre a mesa (Figura 6).

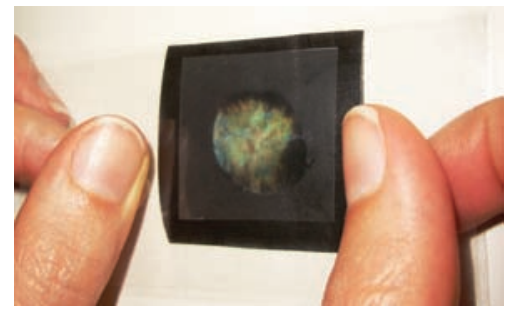

Figura 6. Colocação do adesivo para selagem do dispositivo

f) Retire o adesivo da mesa e dobre as bordas da fita para baixo para selar todos os lados e evitar que a mistura liquido-cristalina extravase.

\section{RESULTADOS E DISCUSSÃO}

As mudanças de cores dos cristais líquidos colestéricos induzidas por temperatura estão diretamente relacionadas a sua estrutura química. Na Figura 7 são mostradas as sete misturas líquido-cristalinas preparadas de acordo com a Tabela 1. Para analisar o efeito procure se fixar na parte superior de cada frasco, onde o material forma um filme fino e melhora a observação das alterações de cor provocadas pelo aumento gradual de temperatura.

Os frascos que aparecem esbranquiçados, na parte superior, indicam que o material está na fase sólida. À medida que são aquecidos, as misturas fundem para a fase líquido-cristalina colestérica. As misturas tornam-se coloridas como resultado do efeito termocrômico. Quando o aquecimento é mantido, as misturas atingem gradualmente as temperaturas de transição entre os estados líquido-cristalino e líquido. Ao atingirem o estado líquido tornamse transparentes.

Como visto no experimento, vários cristais líquidos podem ser misturados para formar misturas que exibem mudanças de cor sobre uma determinada faixa de temperatura.

Este fenômeno pode ser descrito visualizando-se a Figura 8. O fluido líquido-cristalino colestérico funciona como uma mola entre duas placas. Ondas de luz refletida satisfazendo a lei de Bragg emergem em fase e adicionam-se construtivamente, dando origem a uma banda de comprimentos de onda. Essa banda de comprimentos de onda de luz refletida ocorre quando $\Delta \lambda=\mathrm{P} \Delta \mathrm{n}$, onde $\mathrm{P}=2 \mathrm{~d}$ é o passo da hélice, d é a periodicidade espacial e $\Delta \mathrm{n}$ é a birrefringência do cristal líquido. Por outro lado, o passo da hélice $\mathrm{P}$ é função da temperatura. Na maior parte dos derivados colestéricos, $\mathrm{P}$ é uma

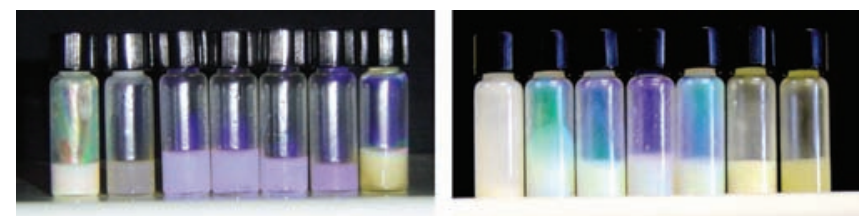

(a) misturas iniciais a $25^{\circ} \mathrm{C}$

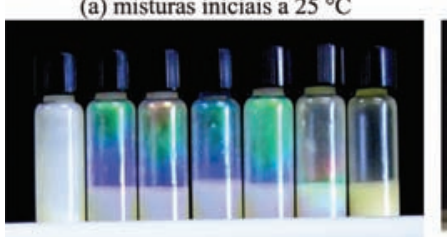

(b) misturas a $28^{\circ} \mathrm{C}$

(c) misturas a $32^{\circ} \mathrm{C}$

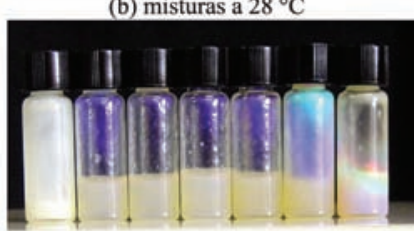

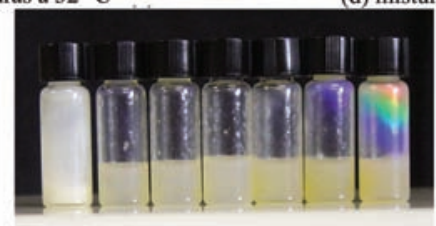

(e) misturas a $40^{\circ} \mathrm{C}$

Figura 7. Da esquerda para a direita são mostradas as sete misturas líquidocristalinas preparadas de acordo com a Tabela 1. Os frascos foram aquecidos de maneira controlada com uma placa de aquecimento com rampa de aquecimento

função decrescente da temperatura, isto é $\mathrm{dP}(\mathrm{T}) / \mathrm{dT}<0$. O aumento do passo da hélice $\mathrm{P}$ com o decréscimo da temperatura pode ser devido a domínios de ordem de curto alcance do tipo esmético. Assim, quando o cristal líquido é resfriado, a mola é elongada (o passo da hélice, Figura 2, é grande) e reflete comprimentos de onda maiores (deslocamento batocrômico). Quando o cristal líquido é aquecido, a mola se comprime e o passo torna-se menor refletindo comprimentos de onda menores (deslocamento hipsocrômico). Em ma-

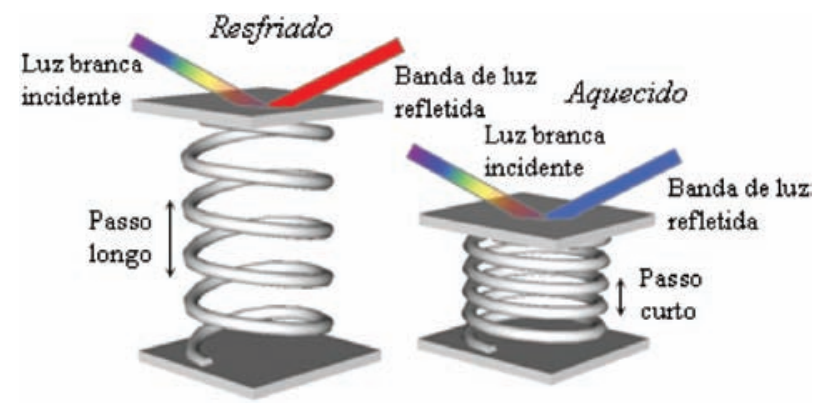

Figura 8. Cristais líquidos colestéricos têm uma estrutura similar a uma mola. Diferentes cores da luz branca ao passarem pelo cristal líquido são refletidas enquanto o material é aquecido

Tabela 1. Misturas de cristais líquidos colestéricos e suas respectivas temperaturas de transição. Diferentes composições fornecem diferentes padrões de cores em diferentes faixas de temperatura

\begin{tabular}{lcccc}
\hline Mistura & $\begin{array}{c}\text { Oleilcarbonato de } \\
\text { colesterila }(\mathrm{g})\end{array}$ & $\begin{array}{c}\text { Pelargonato de } \\
\text { colesterila }(\mathrm{g})\end{array}$ & $\begin{array}{c}\text { Benzoato de } \\
\text { colesterila }(\mathrm{g})\end{array}$ & $\begin{array}{c}\text { Faixa de } \\
\text { transição }\left({ }^{\circ} \mathrm{C}\right)\end{array}$ \\
\hline 1 & 0,70 & 0,10 & 0,20 & $20-25$ \\
2 & 0,45 & 0,45 & 0,10 & $26,5-30,5$ \\
3 & 0,43 & 0,47 & 0,10 & $29-32$ \\
4 & 0,44 & 0,46 & 0,10 & $30-33$ \\
5 & 0,40 & 0,50 & 0,10 & $32-35$ \\
7 & 0,36 & 0,54 & 0,10 & $34-37$ \\
\hline
\end{tabular}




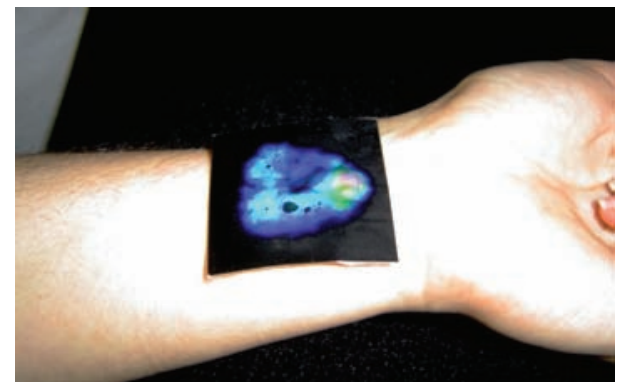

Figura 9. O adesivo preparado no procedimento 2 quando colocado sobre o pulso mostra várias cores

teriais colestéricos que não apresentam domínios esméticos, a situação a relação entre o comprimento do passo helicoidal e temperatura é direta, isto é aumento de temperatura provoca aumento no valor do passo.

Utilizando essa propriedade termocrômica é possível construir um termômetro de cristal líquido ou ainda, um adesivo que mapeie a temperatura de um objeto ou partes do corpo humano. No procedimento 2 da parte experimental foi descrita a preparação de um adesivo de cristal líquido colestérico. Tomando esse adesivo, faça um teste colocando-o sobre a parte interna do pulso, como mostrado na Figura 9.

Regiões que refletem a cor azul indicam pontos de temperaturas mais altas, enquanto regiões que refletem a cor verde são mais frias. $\mathrm{O}$ vermelho, nessa situação, indica a região mais fria do pulso. Se ao invés da mistura líquido-cristalina 7 da Tabela 1 usarmos uma mistura de oleilcarbonato de colesterila e pelargonato de colesterila $(50 \%$ em peso) será obtida uma amostra melhor ainda para explorar a temperatura superficial do corpo humano. Essa mistura varia do vermelho ao azul entre 31,7 e $34,4{ }^{\circ} \mathrm{C}$, conforme Figura 10.

No início dos anos 60 , cientistas usaram cristais líquidos colestéricos para mapear o corpo por causa de variações de temperatura relacionadas com a má circulação sanguínea, tumores ou outras anomalias. Este campo da ciência é chamada de termografia, e imagens hoje em dia são tomadas com câmeras infravermelhas que podem "ver" o calor gerado pelo corpo.

\section{CONCLUSÃO}

Foram descritos experimentos simples capazes de demonstrar importantes propriedades de sistemas auto-organizados quirais. Ao realizar esses experimentos alunos de graduação e pós-graduação são capazes de visualizar alguns efeitos que a quiralidade impõe sobre estruturas super e supramoleculares, em especial cristais lí-

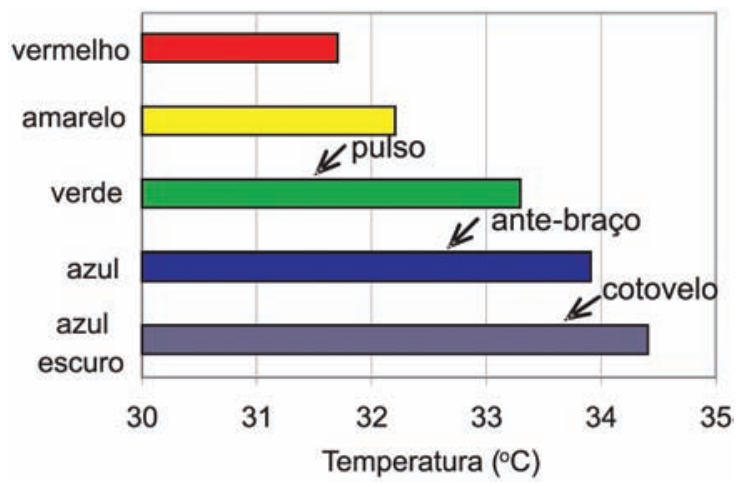

Figura 10. Gráfico ilustrando as cores observadas em um adesivo de cristal líquido, oleilcarbonato de colesterila e pelargonato de colesterila $(50 \% \mathrm{em}$ peso), colocado sobre a superficie do corpo humano. As setas indicam as temperaturas aproximadas para cada parte do braço

quidos. Com o advento da nanotecnologia, onde estruturas desse tipo têm papel relevante, experimentos como os descritos nesse artigo podem se tornar poderosos instrumentos para a familiarização dos estudantes com esse novo ramo da ciência e da tecnologia.

\section{AGRADECIMENTOS}

À FAPESP pela bolsa de pós-doutorado do Dr. F. Ely e ao Prof. H. Gallardo da UFSC pela doação de alguns cristais líquido utilizados nesse trabalho.

\section{REFERÊNCIAS}

1. Kitzerow, H-S.; Bahr, C.; Chirality in Liquid Crystals, Springer: New York, 2001; Kelly, S.M.; O’Neill, M.; Liquid Crystals, Display and Laser Materials, Academic Press.: London, 2000, vol. 7.

2. Grüneberg, K.; Naciri, J.; Shashidhar, R.; Chem. Mater. 1996, 8, 2486.

3. Ely, F.; Tese de Doutorado, Universidade Federal de Santa Catarina, Brasil, 2004; Li, Q.; Li, L.; Kim, J.; Park, H-s.; Williams, J.; Chem. Mater. 2005, 17, 6018; Hikmet, R. A. M.; Kemperman, H.; Nature 1998, 392, 476; Liu, J-H.; Yang, P-C.; Liq. Cryst. 2005, 32, 539.

4. Goodby, J. W.; Demus, D.; Gray, G. W.; Spiess, H. -W.; Vill, V.; Handbook of Liquid Crystals, Wiley-VCH.: Weinheim, 1998, vol. 2B; Colings, P. J.; Patel, J. S.; Handbook of Liquid Crystals Research, Oxford University Press.: New York, 1997.

5. http://plc.cwru.edu/tutorial/enhanced/files/lcd/intro.htm, acessada em Setembro 2006.

6. Heiney, P. A.; Fontes, E.; de Jeu, W. H.; Riera, A.; Carroll, P.; Smith, A. B.; J. Phys. 1989, 50, 461. 